\title{
Dissociating sources of dual-task interference using human electrophysiology
}

\author{
KAREN M. ARNELL, ALICIA M. HELION, JESSICA A. HURDELBRINK, and BRIAN PASIEKA \\ North Dakota State University, Fargo, North Dakota
}

\begin{abstract}
In the psychological refractory period (PRP) paradigm, two unmasked targets are presented, each of which requires a speeded response. Response times to the second target (T2) are slowed when T2 is presented shortly after the first target (T1). Electrophysiological studies have previously shown that the P3 event-related potential component is not delayed during T2 response slowing in the PRP paradigm, but that the lateralized readiness potential is delayed, which suggests a bottleneck on response selection operations but not on stimulus identification. Recently, researchers (Arnell \& Duncan, 2002; Jolicœur \& Dell'Acqua, 1999) observed T2 response slowing in an encoding-speeded response (ESR) paradigm where $\mathrm{T} 2$ followed a masked $\mathrm{T} 1$ that required identification but not a speeded response. T2 response slowing in the ESR paradigm is often indistinguishable from that in the PRP paradigm, prompting some researchersto postulate a common processing bottleneck for the two paradigms. With the use of the ESR paradigm, we observed T2 response slowing and, in contrast to the PRP paradigm, we also observed corresponding P3 delays. The results suggest that dissociable bottlenecks underlie the dual-task costs from the two paradigms.
\end{abstract}

When people attend to two targets presented within half a second of each other, performance on the second target (T2) is typically impaired or delayed. In the psychological refractory period (PRP) paradigm, two unmasked targets are presented at variable target-target stimulus onset asynchronies (SOAs), with each target requiring a speeded response. The target stimuli and tasks are typically very simple, yet results show that response times (RTs) to T2 are often lengthened dramatically when T2 is presented soon after the first target (T1), as compared with when the targets are further apart in time (for a review, see Pashler, 1994). Historically, one popular view has been that the PRP reflects a bottleneck on response selection operations (see, e.g., Pashler, 1994; Welford, 1952), in which the mapping from stimulus identity to the required response can proceed for only one stimulus at a time (for a contradictory model, see Schumacher et al., 1999).

Recently, T2 response slowing at short SOAs has also been observed in an encoding-speeded response (ESR) paradigm, in which $\mathrm{T} 1$ is masked and requires identification for a later response but does not require a speeded response (see, e.g., Arnell \& Duncan, 2002; Jolicœur \& Dell' Acqua, 1998, 1999). For example, if T1 is a masked visual digit and T2 is an unmasked auditory tone, participants may be asked to report the pitch of the T2 tone as soon as possible after its presentation and, at the end of the

This work was supported by a grant from the Neuropsychiatric Research Institute (NRI) to the first author. Correspondence concerning this article should be addressed to K. M. Arnell, Department of Psychology, Brock University, St. Catharines, ON, L2S 3A1 Canada (e-mail: karnell@ brocku.ca). trial, to report the identity of the $\mathrm{T} 1$ digit in an unspeeded manner. The presence of T2 response slowing in the ESR paradigm has led some researchers to postulate that conscious stimulus consolidation and response selection require, at least in part, the same limited capacity processing stages and, therefore, result from the same processing bottleneck(Jolicœur, 1999; Jolicœur \& Dell'Acqua, 1998, 1999). This theory is in contrast to the more traditional view of separate processing limitations for stimulus identification and response selection (Pashler, 1989). Although response slowing appears to be similar in the two paradigms, it is unclear whether the $\mathrm{T} 2$ response slowing observed in the PRP paradigm and that observed in the ESR paradigm result from bottlenecks at the same stage of processing or at different stages.

\section{EVENT-RELATED POTENTIAL INVESTIGATIONS}

For researchers favoring bottleneck models, the results from electrophysiology experiments have been useful in constraining the locus of the proposed bottleneck in the PRP paradigm. The P3 (or P300) event-related potential (ERP) component is sensitive to stimulus identification and categorization manipulations, and it has been suggested by some researchers (e.g., Donchin, 1981) to reflect consolidation into working memory. The P3 is sensitive to the task-defined probability of the appearance of a given stimulus, with a larger $\mathrm{P} 3$ for a rare stimulus than that for a frequent one (Donchin, 1981). All else being equal, subtracting each participant's average waveform on frequent trials from their average waveform on infrequent trials produces $\mathrm{P} 3 \mathrm{~s}$ time-locked to a target that are uncontaminated by the influence of other events or pro- 
cesses. Luck (1998) measured the P3 component timelocked to T2 during the PRP paradigm and showed that the P3 was delayed only slightly at short T1-T2 SOAs, as compared with long ones, despite the large delay in RTs at short, as compared with long, SOAs. Hoffman, Houck, MacMillan, Simons, and Oatman (1985) also reported a dissociation between P3 latency and RT under PRP-like conditions. These results suggest that the response slowing in the PRP paradigm stems primarily from a bottleneck that occurs after the identification and categorization operations.

The lateralized readiness potential (LRP) is related to the preparation of movement for a response, and it is thought by some researchers (e.g., Coles, 1989) to be sensitive to response selection operations but not to response execution operations. Osman and Moore (1993) showed that, as with RTs, the LRP was substantially delayed for T2 at short SOAs in the PRP paradigm. Because the LRP is sensitive to response selection and preparation operations, the discovery of commensurate LRP delays at short SOAs suggests that the response slowing in the PRP paradigm results primarily from a bottleneck at or before response selection or movement preparation. Thus, the ERP findings are consistent with models postulating a bottleneck on processes occurring after stimulus identification and categorization, but at or before response selection.

The goal of the present study was to use both behavioral and electrophysiological data to determine whether a common processing bottleneck underlies the behavioral findings in the ESR (Experiment 1) and PRP (Experiment 2) paradigms or whether dissociable bottlenecks underlie the similar behavioral results.

\section{EXPERIMENT 1}

In Experiment 1, T1 was a masked visual target requiring an unspeeded response, and T2 was an unmasked auditory target requiring a speeded response. T2 RTs were expected to show the usual response slowing at short SOAs (Arnell \& Duncan, 2002; Jolicœur \& Dell' Acqua, 1998, 1999). If T2 response slowing results from a bottleneck that occurs before or at identification and categorization processes, $\mathrm{T} 2$ identification and categorization processes should be delayed, and the $\mathrm{P} 3$ should show delays at short SOAs that are proportional to the group mean RT delays. If, on the other hand, T2 response slowing results from a bottleneck that occurs after identification and categorization processes (e.g., response selection), the P3 should not show robust delays at short SOAs relative to the group mean RT delays. This latter pattern is the one observed by Luck (1998) with the PRP paradigm. Such a finding in the ESR paradigm would support theories postulating a common bottleneck for both paradigms (Jolicœur, 1999; Jolicœur \& Dell'Acqua, 1998, 1999).

\section{Method}

Participants. Fourteen right-handed North Dakota State University undergraduate students ( 8 females) participated for course credit. All participants in this study reported no neurological conditions, normal hearing, and normal (or corrected-to-normal) vision. The data from three of the participants could not be included in the analyses because they produced no clearly discernable P3 in at least one of the three SOA conditions.

Design. The design was a $3(\mathrm{~T} 1-\mathrm{T} 2 \mathrm{SOA}) \times 2$ (T2 pitch) factorial. SOA $(100,200$, or $750 \mathrm{msec}$ ) and T2 pitch (low or high) were within-subjects variables. Levels varied randomly within blocks, so that $\mathrm{T} 2$ was presented at a low pitch on $80 \%$ of the trials and a high pitch on $20 \%$ of the trials, and each SOA occurred equally often for each T2 pitch every 30 trials. Each participant performed 840 experimental trials in one session with a break after 420 trials. ${ }^{1}$

Stimuli. T1 was a randomly selected, visually presented digit (2 or 3), with the two numerals occurring equally often every 30 trials. The digit was presented in bold, 18-point, black Courier New font (approximately $1.1^{\circ}$ of visual angle) in the center of a gray background. Each digit was presented for $60 \mathrm{msec}$ and was immediately replaced by a backward pattern mask presented for $100 \mathrm{msec}$.

T2 was one of three spoken words (cot, yes, or bad) presented at equal amplitudes at either a very low or a very high pitch. Each word was recorded by a female voice and was compressed to $150 \mathrm{msec}$. The sounds were presented with 16 bits of resolution through speakers placed immediately to the left and the right of the monitor.

Procedure and Apparatus. A central fixation spot was presented for $1,000 \mathrm{msec}$ at the start of each trial, followed by a blank interval of 700-1,110 msec. One T1 digit and one T2 spoken word were then presented on each trial, separated by 100, 200, or $750 \mathrm{msec}$. The participants were instructed to identify the digit as it was presented but to delay the $\mathrm{T} 1$ response until prompted at the end of the trial. The participants were told to ignore the identity of the spoken word but to identify its pitch and to report it as quickly as possible after the onset of the word while still being accurate. The participants responded to T2 by pressing the 9 key (for high pitch) and the 0 key (for low pitch) with the index and middle fingers of their right hand. Raised Styrofoam labels assisted the participants in maintaining their finger locations across trials without looking at their hands. The computer prompted the participants to enter their $\mathrm{T} 1$ responses $1,000 \mathrm{msec}$ after their $\mathrm{T} 2$ responses. The participants were told to be accurate (not fast) when making this response. The next trial began $2 \mathrm{sec}$ after the T1 response.

A Dell Pentium II with a 17-in. color monitor, running E-Prime (Schneider, Eschman, \& Zuccolotto, 2002), was used to present stimuli and record behavioral responses. A Neuroscan workstation was used to acquire and analyze electroencepha lographic data recordings from 64 sites (cap by Electrocap International) referenced to the earlobes. Electro-ocular recordings were taken. The signals were amplified with a bandpass of $0.15-100 \mathrm{~Hz}$ and digitized at a rate of $500 \mathrm{~Hz}$. Epochs were created that began $200 \mathrm{msec}$ prior to T2 presentation and ended $1,000 \mathrm{msec}$ after T2 presentation. ${ }^{2}$ Each participant's average waveform on low pitch trials was subtracted from their average waveform on high pitch trials, which produced $\mathrm{P} 3 \mathrm{~s}$ time-locked to $\mathrm{T} 2$ that were uncontaminated by $\mathrm{T} 1$ processing or by any other brain activation that was unaffected by the low versus high manipulation (Luck, 1998). ${ }^{3}$

\section{Results}

Behavior. Mean T2 RTs (high and low pitch trials combined) are presented in Figure $1 \mathrm{~A}$ as a function of T1-T2 SOA. For both experiments, means are from correct T2 trials only (regardless of T1 accuracy). However, the same patterns were observed when T2 RT inclusion was made conditional on the correct report of T1. Less than $4 \%$ of the RTs were removed using Van Selst and Jolicœur's (1994) outlier elimination procedure. A significant effect of SOA was found for T2 RTs $[F(2,20)=$ $30.68, p<.001$ ], showing that T2 RTs increased markedly 

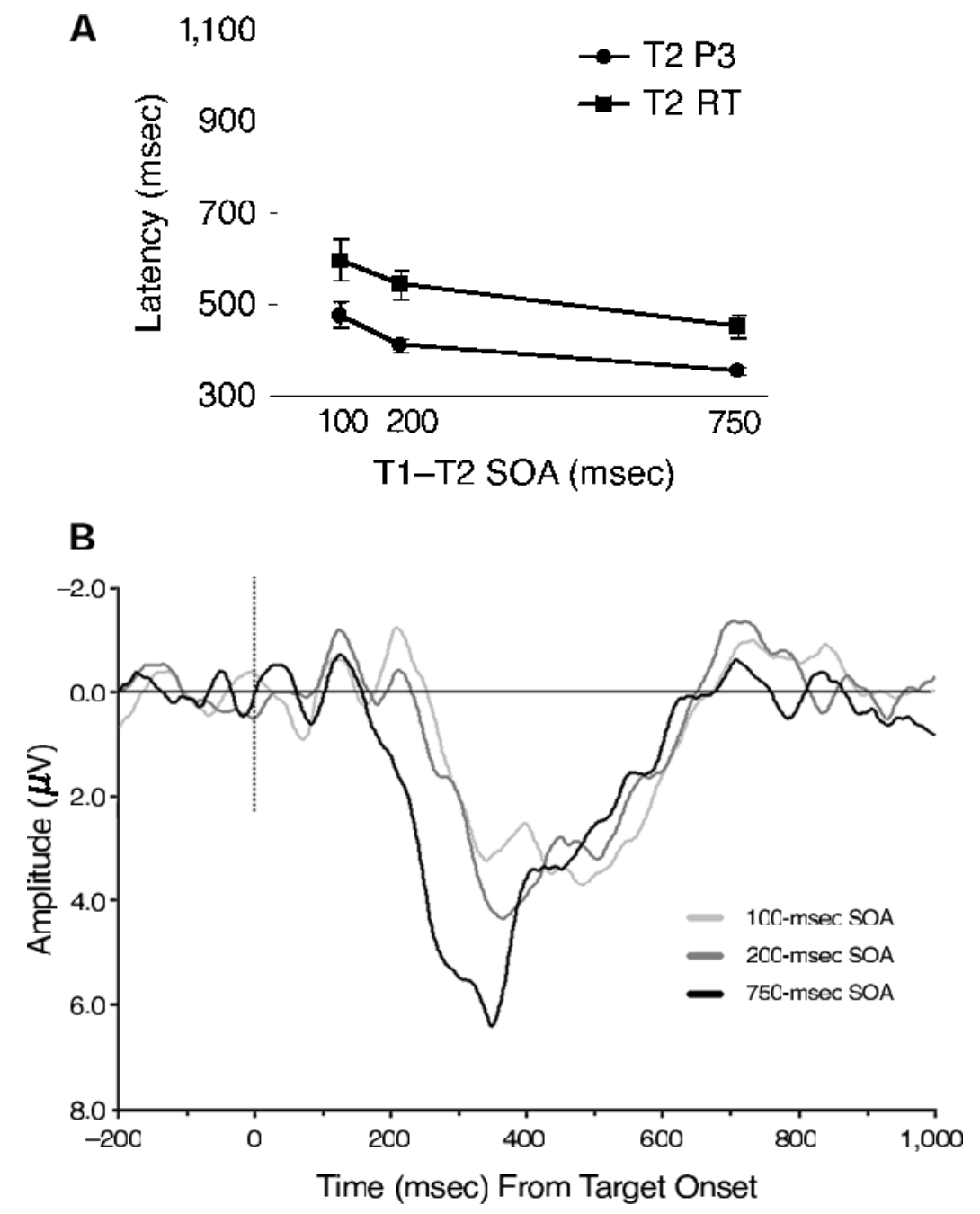

Figure 1. Panel A shows the group mean T2 latency for response times (RTs, squares) and $P 3$ components (circles) from Experiment 1 as a function of stimulus onset asynchrony (SOA) condition $(100,200$, or $750 \mathrm{msec})$. Time on the $x$-axis represents the SOA between $\mathrm{T} 1$ and $\mathrm{T} 2$ in milliseconds. Panel $\mathrm{B}$ shows the P3 subtraction waveforms (high - low pitch) for the three SOA conditions $(100,200$, and $750 \mathrm{msec})$. Time on the $x$-axis represents time from $\mathrm{T} 2$ onset. Following convention, negative is plotted upward.

as SOAs decreased. Paired-sample $t$ tests showed significant mean RT differences for all SOA comparisons $(p \mathrm{~s}<$ .001 for $100-$ vs. $200-\mathrm{msec}, 100-\mathrm{vs} .750-\mathrm{msec}$, and 200 vs. 750 -msec SOAs). T2 accuracy $(94.5 \%, 95.0 \%$, and 95.3\% accuracy for 100-, 200-, and 750-msec SOAs, respectively) did not vary as a function of SOA $(F<1)$. T1 accuracy $(94.5 \%, 94.6 \%$, and $94.8 \%$, respectively) also did not vary significantly with SOA $(F<1)$.

ERPs. Grand-average P3 difference waves, averaged across medial central/parietal electrode sites $(3 \times 4$ grid of electrodes centered on sites $\mathrm{Pz}$ and PzA, where the P3 is typically measured and is often largest), are presented in Figure 1B for each SOA condition. Mean latency of the P3 component is plotted in Figure 1A. A fractional area latency analysis (the latency at which one half of the P3 component's area has been achieved) was used to estimate P3 latency, since this procedure was used by Luck (1998) and is often less susceptible to noise than is peak latency. However, when peak latency (latency of highest positive amplitude) was used as the dependent variable, the same results were observed. ${ }^{4}$ Mean P3 latency varied significantly as a function of SOA $[F(2,20)=9.59, p<$ .005], with longer P3 latencies as SOAs decreased. Paired-samples $t$ tests showed P3 latency differences only for the longest SOA relative to each of the two shorter SOAs $(p \mathrm{~s}<.05)$. 
Mean RT latency and mean P3 latency (from Figure 1A) were analyzed with a $2 \times 3$ within-subjects analysis of variance (ANOVA), with latency measure (RT or P3) and SOA as factors. There was a significant main effect of latency measure, with the overall RT latency longer than than P3 latency $[F(1,10)=12.86, p<.005]$, and a significant main effect of SOA, showing that latency increased as SOA decreased $[F(2,20)=21.69, p<.001]$. Importantly, the latency measure $\times$ SOA interaction did not approach significance $(F<1)$, showing equally large effects of SOA for the two latency measures.

Across participants, the magnitude of the response slowing at short SOAs (the mean RT at the $750-\mathrm{msec}$ SOA subtracted from the mean RT at the 100-msec SOA) was highly correlated $(r=.73)$ with the magnitude of $\mathrm{P} 3$ slowing at short SOAs (the mean P3 latency at the 750msec SOA subtracted from the mean P3 latency at the 100-msec SOA). P3 slowing explained a significant 53\% of the variability $\left(R^{2}=.536\right)$ in response slowing $[t(9)=$ $3.23, p<.01]$. This relationship was observed even when overall baseline $\mathrm{T} 2$ response latencies (overall $\mathrm{T} 2$ RT for each participant) were first partialed out $\left[R^{2}\right.$ change $=.363 ; t(9)=2.51, p<.05]$, providing evidence that the relationship between response slowing and P3 slowing across participants did not result from differences in overall response speed across participants.

P3 amplitude (height of the maximum positivity during the P3) decreased significantly at shorter SOAs $[F(2,20)=4.84, p<.02]$.

\section{Discussion}

As was expected from previous research (Arnell \& Duncan, 2002; Jolicœur \& Dell'Acqua, 1998, 1999), T2 RTs slowed significantly (142 msec) as SOA decreased, even though $\mathrm{T} 1$ was masked and required a subsequent unspeeded response. T2 P3 latencies showed the same pattern (120 msec of P3 slowing). Thus, behavioral responses and the P3 were both delayed as SOAs decreased, and the $\mathrm{P} 3$ slowing was $84.5 \%$ of the size of the response slowing. Furthermore, the amount of P3 slowing was highly and significantly correlated with the amount of response slowing across participants. Because the $\mathrm{P} 3$ is sensitive to the timing of stimulus identification and categorization operations, the observation of $\mathrm{P} 3$ slowing almost as large as response slowing in this experiment provides evidence that the majority of interference underlying the response delay occurred before or at the stage of stimulus identification and categorization.

The present results from the ESR paradigm contrast with those observed by Luck (1998) using the PRP paradigm. Luck observed only minimal P3 slowing relative to RT slowing $(<25 \%)$ and concluded that the interference in the PRP paradigm occurred primarily after stimulus identification and categorization.

\section{EXPERIMENT 2}

The different patterns of results observed in Experiment 1 and in Luck's (1998) study may reflect separate sources of interference in the ESR and the PRP paradigms, or they may have resulted from methodological differences. In Experiment 2, we replicated Experiment 1, except that T1 was changed so that Experiment 2 employed a PRP paradigm (i.e., the T1 mask was removed and a speeded forced-choice response was required). If the contrasting results are due to dissociable processing bottlenecks in the PRP and the ESR paradigms, we should replicate Luck's results, and the grand average P3 in Experiment 2 should not show delays at short SOAs that are proportional to the group mean RT delays. If, however, the contrasting results are due to methodological differences and not to dissociable processing bottlenecks, the grand average $\mathrm{P} 3$ should continue to show robust delays at short SOAs relative to the group mean T2 RT delays.

\section{Method}

Participants. Thirteen right-handed North Dakota State University undergraduate students ( 7 females) participated for course credit. Data from three of the participants could not be analyzed because they produced no clearly discernable P3 in at least one of the three SOA conditions.

Stimuli and Procedure. Experiment 2 used the same stimuli, design, and procedures as those in Experiment 1, except as specified below. T1 was again presented for $60 \mathrm{msec}$, but this time no mask trailed its presentation. The participants were instructed to make a speeded response indicating whether the digit was a 2 or a 3 using the middle ( 2 key) and index ( 3 key) fingers, respectively, of their left hands and to make a speeded response indicating whether the T2 word was low or high in pitch using the index (9 key) and middle ( 0 key) fingers, respectively, of their right hands. No order constraints were placed on the responses. Owing to movement artifacts, ocular artifacts, or amplifier saturation, between $3 \%$ and $28 \%$ of the trials per participant were removed prior to averaging, as above.

\section{Results}

Behavior. Mean T2 RTs (high and low pitch trials combined) are presented in Figure 2A as a function of T1T2 SOA. Less than $4 \%$ of the trials were removed as outliers. A significant effect of SOA was observed $[F(2,18)=$ 52.32, $p<.001$ ], showing that T2 RTs increased markedly as SOA decreased. Paired-sample $t$ tests showed significant mean RT differences for all SOA comparisons (all $\left.p_{\mathrm{s}}<.001\right)$. T2 accuracy $(93.3 \%, 93.3 \%$, and $94.3 \%$ accuracy for 100-, 200-, and 750-msec SOAs, respectively) did not vary as a function of SOA $(F<1)$.

Mean T1 RTs showed a significant effect of SOA $[F(2,18)=8.18, p<.01]$, because RTs increased at longer SOAs $(786,810$, and 1,041 msec at 100-, 200-, and 750-msec SOAs, respectively). Mean digit accuracy $(97.8 \%, 97.8 \%$, and $97.9 \%$ accuracy at $100-, 200-$, and 750-msec SOAs, respectively) did not vary as a function of SOA $(F<1)$. This pattern of RTs for the two targets suggests that at least some participants may have grouped their responses on some proportion of trials and that the magnitude of the $\mathrm{T} 2$ response slowing may be somewhat underestimated.

ERPs. Grand-average P3 difference waves from the same medial central/parietal electrode sites as those in Experiment 1 are presented in Figure 2B for each SOA condition. Mean latency of the P3 is plotted in Fig- 

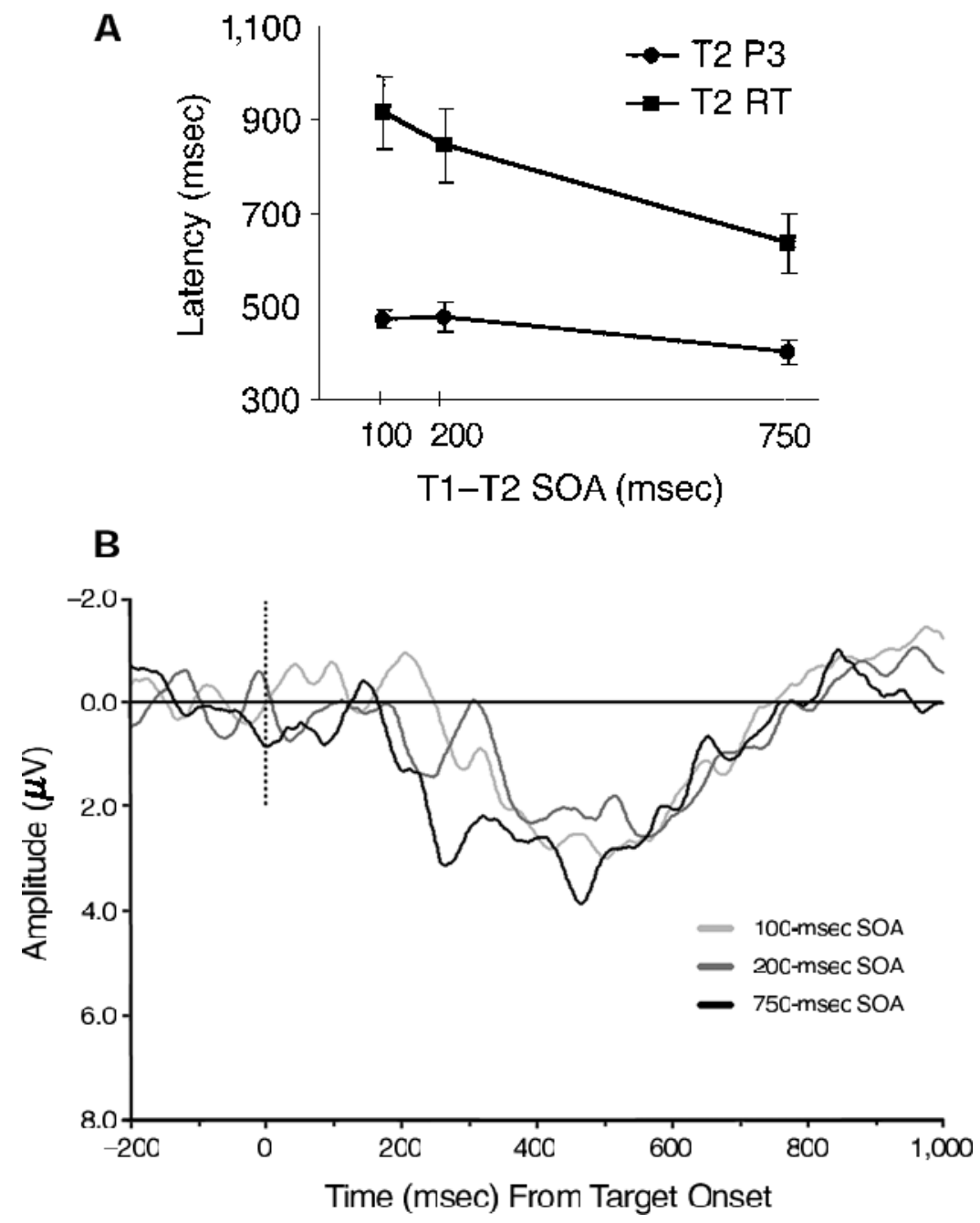

Figure 2. Panel A shows the group mean T2 latency for response times (RTs, squares) and $P 3$ components (circles) from Experiment 2 as a function of stimulus onset asynchrony (SOA) condition $(100,200$, or $750 \mathrm{msec})$. Time on the $x$-axis represents the SOA between T1 and T2 in milliseconds. Panel B shows the P3 subtraction waveforms (high - low pitch) for the three SOA conditions $(100,200$, and $750 \mathrm{msec})$. Time on the $x$-axis represents time from $\mathrm{T} 2$ onset. Following convention, negative is plotted upward.

ure 2A. P3 latency did vary significantly as a function of SOA $[F(2,18)=7.71, p<.01]$, with longer latencies at shorter SOAs. ${ }^{5}$ Paired-samples $t$ tests showed P3 latency differences only for the longest SOA relative to both shorter SOAs $(p \mathrm{~s}<.01)$.

Mean RT latency and mean P3 latency (from Figure $2 \mathrm{~A}$ ) were also analyzed with a $2 \times 3$ within-subjects ANOVA. There was a significant main effect of latency measure, with the overall RT latency longer than the P3 latency $[F(1,9)=15.92, p<.005]$, and a significant main effect of SOA, in which latency increased as SOA decreased $[F(2,18)=53.38, p<.001]$. Importantly, the latency $\times$ SOA interaction was also significant $[F(2,18)=$ $18.77, p<.001$ ], owing to the larger SOA effect for RT latencies than for P3 latencies.
The magnitude of the response slowing at short SOAs was not highly correlated $(r=.22)$ with the magnitude of P3 slowing at short SOAs. P3 slowing explained a nonsignificant $5 \%$ of the variability $\left(R^{2}=.049\right)$ in response slowing $(F<1)$, and this was also true when overall baseline T2 RTs were first partialed out.

P3 amplitude decreased significantly as SOA decreased $[F(2,18)=6.19, p<.01]$.

\section{Discussion}

Although significant, the magnitude of the overall P3 slowing was relatively small (69 msec) and was only $25 \%$ of the size of the response slowing $(278 \mathrm{msec})$. Furthermore, the amount of $\mathrm{P} 3$ slowing was not significantly related to the amount of response slowing across partici- 
pants. The relatively small amount of $\mathrm{P} 3$ slowing at short SOAs indicates that stimulus identification and categorization operations were only slightly delayed at short SOAs, and that the majority of interference underlying the $\mathrm{T} 2$ response delay in this experiment occurred after stimulus identification and categorization operations. These results replicate those of Luck (1998).

\section{GENERAL DISCUSSION}

In replication of previous behavioral results (Arnell \& Duncan, 2002; Jolicœur \& Dell'Acqua, 1998, 1999), T2 response slowing was observed when $\mathrm{T} 1$ was masked and required a delayed unspeeded response (Experiment 1) and when T1 was unmasked and required an immediate speeded response (Experiment 2). The response slowing observed in the ESR and the PRP paradigms is often indistinguishable. ${ }^{6}$ This has led some researchers (e.g., Jolicœur, 1999; Jolicœur \& Dell'Acqua, 1999) to suggest that the response slowing observed in both paradigms results from a processing bottleneck common to speeded response selection and stimulus consolidation into working memory.

In contrast, the present results suggest that there are two separate processing bottlenecks: one for conscious stimulus identification (and/or consolidation) and one for response selection. ${ }^{7}$ In the ESR paradigm, conscious stimulus identification is bottlenecked given that both targets need to be identified "on-line." However, a bottleneck on response selection operations is not encountered in this paradigm, given that $\mathrm{T} 1$ does not require a speeded response. In Experiment 1, the P3 latency delay observed with decreasing T1-T2 SOAs was robust and almost equal to the response slowing, which provides evidence that the interference resulted primarily from a processing bottleneck at or before stimulus identification and categorization. Little, if any, interference was observed after this stage, given that response selection could proceed unimpaired.

However, in the PRP paradigm, both stimulus identification and response selection operations are bottlenecked, given that both $\mathrm{T} 1$ and $\mathrm{T} 2$ require identification and response selection. In Experiment 2, there was a modest, yet significant, P3 latency delay at short SOAs, which provides evidence for some interference at or before stimulus identification. However, in Experiment 2 the small amount of $\mathrm{P} 3$ slowing contrasted with the large response slowing, which provides evidence that a large amount of interference resulted from a processing bottleneck after stimulus identification and categorization operations-likely on response selection operations. According to this logic, RT delays at short SOAs in the ESR paradigm result entirely from processing delays on stimulus identification, whereas RT delays at short SOAs in the PRP paradigm result from a combination of processing delays on stimulus identification and response selection.

The amount of observed P3 slowing was numerically larger in Experiment $1(120 \mathrm{msec})$ than in Experiment 2
$(69 \mathrm{msec})$. However, an experiment $(1,2) \times \operatorname{SOA}(100$, 200 , and $750 \mathrm{msec}$ ) ANOVA on P3 latency produced an interaction that was only marginally significant $(p<.10)$, making it unclear whether this difference is meaningful. If real, the possibly larger P3 latency delay in Experiment 1 may have resulted from T1's occupation of the bottlenecked identification stage for a longer time when T1 was masked (Experiment 1) relative to when it was unmasked (Experiment 2). Alternatively, because of the need for off-line report in Experiment 1, T1 may have required consolidation in working memory (Jolicœur, 1999) in addition to stimulus identification, and this additional processing may have increased the P3 delay in the ESR paradigm. This consolidation may have been unnecessary in Experiment 2, in which T1 required a speeded response. This difference could also underlie the possible dissimilarities in P3 latency delay across experiments.

The proposition of a bottleneck in the ESR paradigm during the identification stage is consistent with ERP research in which T2 required an unspeeded response. Vogel and Luck (2002) showed P3 latency delays at short T1-T2 SOAs when T1 was masked and T2 was unmasked but both required an unspeeded response. Their results suggest that identification of a masked $\mathrm{T} 1$ results in identification and categorization delays for $\mathrm{T} 2 \mathrm{~s}$ presented at short SOAs. However, when both T1 and T2 are masked and require an unspeeded response, $\mathrm{T} 2$ identification cannot be delayed because T2 will be overwritten by its mask (Giesbrecht \& Di Lollo, 1998). Under such conditions, T2 accuracy suffers at short SOAs (the attentional blink; Raymond, Shapiro, \& Arnell, 1992), and P3 amplitude is dramatically reduced (Vogel, Luck, \& Shapiro, 1998).

Even in the present experiments in which T2 was unmasked, P3 amplitude was reduced somewhat at short SOAs, replicating Luck's (1998) and Hoffman et al.'s (1985) results. Indeed, Hoffman et al. suggested that P3 amplitude reflected the amount of a limited capacity resource that is separate from a resource concerned with motor responses, which is not unlike the separate bottleneck model proposed here.

It is currently unclear whether the different patterns of results in Experiment 1 and Experiment 2 are due the presence or absence of T1 masking, the presence or absence of T1 speeded responding, or both, given that these were purposely confounded in the present set of experiments. It is also unclear why $\mathrm{T} 1$ response selection demands appear to influence the identification of a masked $\mathrm{T} 2$ if identification of a masked $\mathrm{T} 1$ does not influence response selection for T2 (Arnell \& Duncan, 2002). Investigations continue in our laboratory and may yield further insights on these issues. However, the present results suggest that the dualtask interference observed in the ESR and PRP paradigms do not result from a single common processing bottleneck.

\section{REFERENCES}

ARNELL, K. M., \& DunCAN, J. (2002). Separate and shared sources of dual-task cost in stimulus identification and response selection. Cognitive Psychology, 44, 105-147. 
Coles, M. G. H. (1989). Modern mind-brain reading: Psychophysiology, physiology and cognition. Psychophysiology, 26, 251-269.

Dell'ACQua, R., JolicÄUR, P., Pesciare Lli, F., Job, R., \& PAlomba, D. (2003). Electrophysiological evidence of visual encoding deficits in a cross-modal AB paradigm. Psychophysiology, 40, 629-639.

Donchin, E. (1981). Surprise! . . . Surprise? Psychophysiology, 18, 493-513.

Giesbrecht, B. L., \& Di Lollo, V. (1998). Beyond the attentional blink: Visual masking by item substitution. Journal of Experimental Psychology: Human Perception \& Performance, 24, 1454-1466.

Hoffman, J. E., Houck, M. R., MacMillan, F. W., Simons, R. F., \& OATMAN, L. C. (1985). Event-related potentials elicited by automatic targets: A dual-task analysis. Journal of Experimental Psychology: Human Perception \& Performance, 11, 50-61.

JOLICÄUR, P. (1999). Concurrent response selection demands modulate the attentional blink. Journal of Experimental Psychology: Human Perception \& Performance, 25, 1097-1113.

JolicÄUR, P., \& Dell'ACQUA, R. (1998). The demonstration of shortterm consolidation. Cognitive Psychology, 36, 138-202.

JolicÄUR, P., \& DELL'ACQUA, R. (1999). Attentional and structural constraints on memory encoding. Psychological Research, 62, 154-164.

LUCK, S. J. (1998). Sources of dual-task interference: Evidence from human electrophysiology. Psychological Science, 9, 223-227.

OSMAn, A., \& MoORE, C. M. (1993). The locus of dual-task interference: Psychological refractory effects on movement-related brain potentials. Journal of Experimental Psychology: Human Perception \& Performance, 19, 1292-1312.

PASHLER, H. (1989). Dissociations and dependencies between speed and accuracy: Evidence for a two-component theory of divided attention in simple tasks. Cognitive Psychology, 21, 469-514.

PASHLER, H. (1994). Dual-task interference in simple tasks: Data and theory. Psychological Bulletin, 116, 220-244.

RAYMOND, J. E., SHAPIRO, K. L., \& ARNELL, K. M. (1992). Temporary suppression of visual processing in an RSVP task: An attentional blink? Journal of Experimental Psychology: Human Perception \& Performance, 18, 849-860.

Schneider, W., Eschman, A., \& Zuccolotto, A. (2002). E-prime user's guide. Pittsburgh, PA: Psychology Software Tools Inc.

Schumacher, E. H., Lauber, E. J., Glass, J. M., Zurbriggen, E. L., GmeindL, L., Kieras, D. E., \& MeYer, D. E. (1999). Concurrent response-selection processes in dual-task performance: Evidence for adaptive executive control of task scheduling. Journal of Experimental Psychology: Human Perception \& Performance, 25, 791-814.

VAN SELST, M., \& JOLICÄUR, P. (1994). A solution to the effect of sample size and skew on outlier elimination. Quarterly Journal of Experimental Psychology, 47A, 631-650.

VOGEL, E. K. \& LUCK, S. J. (2002). Delayed working memory consolidation during the attentional blink. Psychonomic Bulletin \& Review, 9, 739-743.
Vogel, E. K., LuCK, S. J., \& SHAPIRo, K. L. (1998). Electrophysiological evidence for a postperceptual locus of suppression during the attentional blink. Journal of Experimental Psychology: Human Perception \& Performance, 24, 1656-1674.

WELFORD, A. T. (1952). The "psychological refractory period" and the timing of high-speed performance: A review and theory. British Journal of Psychology, 43, 2-19.

\section{NOTES}

1. The purpose of the present study was to investigate the underlying sources of the dual-task costs observed in the PRP and the ESR paradigms. Previous studies in which these two paradigms were compared have used unpracticed naive participants, and therefore we have done the same.

2. Owing to movement artifacts, ocular artifacts, or amplifier saturation, between $5 \%$ and $34 \%$ of trials per participant were removed prior to averaging. Over $90 \%$ of the removed trials were due to blinks during the epoch. All trials in which the VEOG recording showed a large ( $>$ approximately $25 \mu \mathrm{v}$ ) change from the $0 \mu \mathrm{v}$ baseline, according to visual inspection by the experimenter, were removed.

3. Prior to subtraction, each pair of high and low pitch waveforms had highly similar onsets and P3 latencies. Thus, the subtraction did not create a P3 peak latency unrepresentative of either of the waveforms from which it was derived.

4. P3 peak latency (time from T2 onset until peak amplitude was achieved) also varied significantly as a function of $\operatorname{SOA}[F(2,20)=$ $6.20, p<.01]$ and showed no measure $\times$ SOA interaction when analyzed with T2 RTs $(F<1)$, just as was found for the fractional area latency measure. P3 slowing was estimated as $127 \mathrm{msec}$ when using peak latency and as $120 \mathrm{msec}$ when using fractional area.

5. $\mathrm{P} 3$ peak latency also varied significantly with $\operatorname{SOA}[F(2,18)=$ $3.56, p<.05]$. Furthermore, peak latency showed a significant latency $\times$ SOA interaction when analyzed with T2 RTs $[F(2,18)=12.51$, $p<.001]$, as did the fractional area measure. P3 slowing was estimated as $71 \mathrm{msec}$ when using peak latency and as $69 \mathrm{msec}$ when using fractional area.

6. Although the RT slope is steeper in Experiment 2 than in Experiment 1 , the relative size of the effect alone cannot generally distinguish the paradigm or the nature of the interference.

7. Dell'Acqua, Jolicœur, Pesciarelli, Job, and Palomba (2003) have recently observed that T2 P3 amplitude and response accuracy were both reduced at short SOAs with an unmasked speeded $\mathrm{T} 1$ and a masked speeded $\mathrm{T} 2$, but only when $\mathrm{T} 1$ required a three-alternative, not a two-alternative, forced-choice response. Their results suggest that response selection may interfere with $\mathrm{T} 2$ stimulus consolidation under some conditions.

(Manuscript received March 27, 2002; revision accepted for publication December 6, 2002.) 